\title{
Sequential butylphthalide therapy combined with dual antiplatelet therapy in the treatment of acute cerebral infarction
}

\author{
Xuewen Wo ${ }^{1}$, Jinyan $\mathrm{Han}^{2}$, Jiajia Wang ${ }^{3}$, \\ Xinmin Wang ${ }^{4}$, Xiaoying Liu ${ }^{5}$, Zhonggong Wang ${ }^{6}$
}

\section{ABSTRACT}

Objective: To observe the clinical efficacy of sequential butylphthalide therapy combined with dual antiplatelet therapy in the treatment of elderly patients with acute cerebral infarction $(\mathrm{ACl})$.

Methods: One hundred and twenty-two elderly patients with $\mathrm{ACl}$ who were admitted to the department of neurology of our hospital at May 2016-August 2018 were selected grouped into a control group and an observation group by random number table method, 61 in each group. On the basis of conventional treatment, the patients in the control group were given dual antiplatelet therapy (aspirin enteric-coated tablets + clopidogrel bisulfate tablets), while the patients in the observation group were given sequential butylphthalide therapy on the basis of the control group. The clinical effects of the two groups were compared after four weeks of treatment, and the changes of National Institutes of Health Stroke Scale (NIHSS), ADL score, plasma 3-mercaptopyruvate sulphurtransferase (3-MST) and Amyloid $B_{42}\left(A B_{42}\right)$ levels and the occurrence of adverse reactions during treatment were recorded.

Results: The clinical efficacy of the observation group was better than that of the control group $(\mathrm{P}<0.05)$. There was no significant difference in NIHSS and ADL scores between the two groups before treatment $(P>0.05)$. After treatment, the NIHSS and ADL scores of the observation group were better than those of the control group $(P<0.05)$. There was no significant difference in plasma levels of 3-MST and AB42 between the two groups before treatment $(P>0.05)$. The level of plasma 3-MST in the observation group was higher than that in the control group, and the level of plasma AB42 was lower than that in the control group $(P<0.05)$. No serious adverse reactions occurred during the treatment period in both groups.

Conclusion: Butylphthalide sequential therapy combined with dual antiplatelet therapy is effective in the treatment of elderly $\mathrm{ACl}$. It can effectively improve the plasma level of 3-MST and decrease the plasma level of $A B 42$, which is conducive to improving the living ability and neurological function of patients and has high safety.

KEYWORDS: Acute cerebral infarction, Butylphthalide, Dual antiplatelet, Sequential therapy.

How to cite this:

doi: https://doi.org/10.12669/pjms.36.4.1831

Wo X, Han J, Wang J, Wang X, Liu X, Wang Z. Sequential butylphthalide therapy combined with dual antiplatelet therapy in the treatment of acute cerebral infarction. Pak J Med Sci. 2020;36(4):615-620. doi: https://doi.org/10.12669/pjms.36.4.1831

This is an Open Access article distributed under the terms of the Creative Commons Attribution License (http://creativecommons.org/licenses/by/3.0), which permits unrestricted use, distribution, and reproduction in any medium, provided the original work is properly cited.

Correspondence:

Zhonggong Wang,

Binzhou People's Hospital,

Shandong, No. 515 Huanghe $7^{\text {th }}$ Road,

256610, China.

Email: wangzhonggongdi@163.com

* Received for Publication:

* Revision Received:

* Revision Accepted:
October 16, 2019

April 9, 2020

April 11, 2020

\section{INTRODUCTION}

Acute cerebral infarction (ACI) is a common neurological disease. The incidence of $\mathrm{ACI}$ has been increasing in recent years. ${ }^{1} \mathrm{ACI}$, also known as ischemic stroke, is caused by blood supply disorders induced by various reasons in regional brain tissue, leading to necrosis of ischemic and hypoxic lesions in brain tissues and the clinical 
neurological deficits, ${ }^{2,3} \mathrm{ACI}$ may cause limb function and language dysfunction and can also affect cognitive function of patients in varying degrees, manifested as mental and behavioral abnormalities, apraxia, agnosia, loss of understanding and so on, and it will cause serious harm to patients' lives and living quality. ${ }^{4,5}$

ACI patients are often treated symptomatically by expanding blood volume, reducing intracranial pressure, anticoagulating and controlling basic diseases. However, in recent years, with the change of people's lifestyle, the etiology of ACI is complex and diverse, and the effect of conventional treatment is poor. ${ }^{6,7}$ At present, thrombolysis and anti-platelet aggregation are commonly used in the treatment of ACI patients, and promoting the recovery of patients' nervous function is one of the key points in the treatment of ACI. 8,9 Aspirin combined with clopidogrel is the standard dual antiplatelet therapy scheme for ACI patients. ${ }^{10}$ Butylphthalide is the ACI treatment drug recommended by the Chinese Guidelines for the Diagnosis and Treatment of Acute Ischemic Stroke (2014). ${ }^{11}$ The latest research suggests that butylphthalide can significantly improve the neurological deficits of patients and improve their living ability. ${ }^{12}$

The purpose of this study was to observe the clinical efficacy of butylphthalide injection combined with dual antiplatelet therapy in elderly patients with $\mathrm{ACI}$, in order to provide a reference for clinical effective treatment of ACI.

\section{METHODS}

General data: This is a retrospective study, and the size of sample was calculated using the following formula:

$$
n=2 *[(\alpha \beta) \sigma / \delta] \wedge 2 \text {, }
$$

where $\delta$ stands for the required degree of distinction, $\sigma$ stands for the standard deviation or its estimated value, $s, \alpha$ and $\beta$ can refer to the row of degree of freedom $v=\infty$ - in the table of $t$ critical value.

One hundred and twenty-two elderly patients with $\mathrm{ACI}$ who were admitted to the Department of Neurology of our hospital at May 2016-August 2018 were selected according to the principle of randomization after the approval of Ethical Committee at October 15, 2019. All the patients met the diagnostic criteria of ACI in the Guidelines for Secondary Prevention of Ischemic Stroke and Transient Ischemic Attack of China (2014) and were diagnosed by imaging examination. ${ }^{13}$ All the patients were divided into a control group and an observation group by random number table method, 61 in each group. In the control group, there were 38 males and 23 females, with an average age of $(65.31 \pm 7.56)$ years, and the average time between onset and admission was $(24.51 \pm 4.09) \mathrm{h}$. As to complications, 30 cases had hypertension, 26 cases had diabetes and 33 cases had hyperlipidemia. In the observation group, there were 36 males and 25 females, with an average age of $(63.63 \pm 6.56)$ years, and the average time between onset and admission was $(25.72 \pm 4.81) \mathrm{h}$. As to complications, 35 cases had hypertension, 25 cases had diabetes, and 30 cases had hyperlipidemia. There were no significant differences in gender, age, time between onset and admission, and underlying diseases between the two groups $(\mathrm{P}>0.05)$; therefore, the results were comparable. The research program was approved by the Medical Ethics Committee of the hospital, and the patients or their families signed consent agreement.

Inclusion criteria: Inclusive criteria included aging from 45 to 70 years old, cerebral infarction patients within onset within 6 24 h, and 5 25 points of National Institutes of Health Stroke Scale (NIHSS).

\section{Exclusion Criteria:}

1. Having serious basic diseases in the cardiac, pulmonary, liver and kidney, intracranial hemorrhage, malignant tumors or severe consciousness disorders.

2. Having coagulation dysfunction or bleeding tendency, or taking anticoagulant drugs.

3. Having allergic history.

4. Pregnant or lactating women.

Therapeutic methods: Both groups were given conventional treatment after admission, including oral administration of $20 \mathrm{mg} / \mathrm{d}$ atorvastatin. Patients in the control group were given dual antiplatelet therapy on the basis of conventional treatment: orally taking aspirin enteric-coated tablets (Bayer SFDA approval number J20130078), $200 \mathrm{mg}$ each time, once a day; orally taking clopidogrel bisulfate tablets (Sanofi (Hangzhou) SFDA approval number J20130083), $75 \mathrm{mg}$ each time, once a day.

On the basis of the control group, patients in the observation group were intravenously injected with butylphthalide injection (Enbipu Pharmaceutical Co., Ltd., Shiyao Group, SFDA approval number H20100041), $100 \mathrm{~mL} /$ time, 2 times/day. Two weeks later, the patients 
Treatment of acute cerebral infarction

Table-I: Clinical effects between two groups [n (\%)].

\begin{tabular}{lcccc}
\hline Group & Markedly effective & Effective & Invalid & Effective rate \\
\hline Observation group & $44(72.13)$ & $12(19.67)$ & $5(8.20)$ & $56(91.80)$ \\
Control group & $34(55.74)$ & $14(22.95)$ & $13(21.31)$ & $48(78.69)$ \\
$X^{2}$ & $/$ & $/$ & $/$ & 10.161 \\
$\mathrm{P}$ & $/$ & $/$ & $/$ & $<0.05$ \\
\hline
\end{tabular}

were given butylphthalide capsule (Enbipu Pharmaceutical Co., Ltd., Shiyao Group, SFDA approval number H20050299), $0.2 \mathrm{~g} /$ time, 3 times/day. Both groups were treated for four weeks.

Observation index: The clinical efficacies of two groups of patients were observed. Criteria for judging clinical efficacy included: marked effect: NIHSS score decreased by higher than $90 \%$ after treatment; ${ }^{14}$ effective: $18 \% \leq$ decrease of NIHSS score $<90 \%$ after treatment; ineffective: NIHSS score decreased by lower than 18\%; effective rate $=($ number of cases of marked effect + number of effective cases)/total number of cases $\times 100 \%$. The neurological function of patients was tested and evaluated using NIHSS. The lower the NIHSS score, the milder the neurological functional deficit. The ability of daily living was systematically evaluated using ADL scale (14 items). The higher the score, the more ideal the ability of daily living. $5 \mathrm{ml}$ of fasting peripheral venous blood was taken from each patient before and after treatment, added with heparin sodium anticoagulation, and centrifuged at $3000 \mathrm{r} / \mathrm{min}$ for 10 minutes. The plasma was collected and stored at $-80^{\circ} \mathrm{C}$ for testing. The levels of plasma 3-mercaptopyruvate sulphurtransferase (3-MST) and Amyloid $\beta_{42}\left(\mathrm{~A} \beta_{42}\right)$ were detected by enzyme-linked immunosorbent assay (ELISA) and Pulang DNM-9602G microplate reader. The kit was purchased from Shanghai Jianglai Biological Technology Co., Ltd. and operated strictly according to the kit instructions. The adverse reactions of the two groups during treatment were recorded.

Statistical methods: SPSS 21.0 was used for data processing. The measurement data were expressed as (Mean $\pm \mathrm{SD}$ ). The comparison between groups used two independent-sample $t$ test. The counting data was expressed by relative number and processed by $\mathrm{X}^{2}$ test. The rank sum test was used for ranked data analysis. The difference was assessed as statistically significant if $\mathrm{P}<0.05$.

\section{RESULTS}

The effective rate of treatment in the observation group was $92.5 \%$, which was significantly higher than that in the control group (78.0\%). There was significant difference between the two groups $(\mathrm{P}<0.05$, Table-I).Before treatment, there was no significant difference in NIHSS score and ADL score between the two groups $(\mathrm{P}>0.05)$. After treatment, the improvement of NIHSS and ADL scores in the observation group were significantly better than that in the control group, and the differences were statistically significant $(\mathrm{P}<0.05$, Table-II).

There were no significant differences in plasma levels of 3-MST and $A \beta_{42}$ between the two groups before treatment $(\mathrm{P}>0.05)$. After treatment, the plasma level of 3-MST in the observation group was higher than that in the control group, and the plasma level of $\mathrm{A} \beta_{42}$ was lower than that in the control group; the differences were statistically significant $(\mathrm{P}<0.05$, Table-III).

Table-II: NIHSS score and ADL score between two groups before and after treatment.

\begin{tabular}{lcccc}
\hline \multirow{2}{*}{ Group } & \multicolumn{2}{c}{ NIHSS score } & \multicolumn{2}{c}{ ADL score } \\
\cline { 2 - 5 } & Before treatment & After treatment & Before treatment & After treatment \\
\hline Observation group & $12.7 \pm 5.9$ & $5.9 \pm 3.7$ & $15.6 \pm 6.3$ & $52.3 \pm 14.8$ \\
Control group & $12.8 \pm 3.4$ & $8.6 \pm 4.1$ & $15.6 \pm 5.2$ & $32.6 \pm 12.2$ \\
$\mathrm{t}$ & 0.423 & 6.931 & 0.374 & 8.312 \\
$\mathrm{P}$ & $>0.05$ & $<0.05$ & $>0.05$ & $<0.05$ \\
\hline
\end{tabular}


Xuewen Wo et al.

Table-III: Plasma levels of 3-MST and A $\beta 42$ between two groups before and after treatment.

\begin{tabular}{lcccc}
\hline \multirow{2}{*}{ Group } & \multicolumn{2}{c}{$3-M S T(\mu g / \mathrm{L})$} & \multicolumn{2}{c}{ A beta $42(\mathrm{ng} / \mathrm{L})$} \\
\cline { 2 - 5 } & Before treatment & After treatment & Before treatment & After treatment \\
\hline Observation group & $1.70 \pm 0.23$ & $4.45 \pm 0.18$ & $59.72 \pm 5.14$ & $30.66 \pm 3.63$ \\
Control group & $1.65 \pm 0.21$ & $3.06 \pm 0.35$ & $60.40 \pm 5.26$ & $42.51 \pm 4.83$ \\
$\mathrm{t}$ & 1.217 & 7.671 & 0.703 & 12.917 \\
$\mathrm{P}$ & $>0.05$ & $<0.05$ & $>0.05$ & $<0.05$ \\
\hline
\end{tabular}

During the intervention, there were four cases of significantly increased transaminase $(<80$ $\mathrm{U} / \mathrm{L}$ ), two cases of abdominal discomfort, and one case of nausea in the control group, and the incidence of adverse reaction was $11.48 \%$. In the observation group, there were four cases of significantly increased transaminase $(<80 \mathrm{U} / \mathrm{L})$, two cases of abdominal discomfort and two cases of nausea, and the incidence of adverse reactions was $13.11 \%$. There was no significant difference in the incidence of adverse reactions between the two groups $\left(X^{2}=0.29, P=0.59\right)$. All the above adverse reactions recovered normally after one week of drug withdrawal. All the patients had no adverse reactions such as gastrointestinal bleeding, subcutaneous hemorrhage, coagulation disorders and drug allergy.

\section{DISCUSSION}

The onset of cerebral infarction is rather acute and without warning. After the occurence of cerebral infarction, serious blood circulation disorders will occur in brain tissue in a short time, leading to hypoxia and ischemia in brain tissue. ${ }^{15}$ Therefore, early recovery of cerebral blood supply and nerve function is the key to the treatment of ACI. Anticoagulation, thrombolysis and protection of nerve function are the main therapies. However, the best time window for thrombolysis in ACI patients is within 24 hours after onset. Most patients have missed the best time window for thrombolysis when they see a doctor; at that time, antiplatelet aggregation therapy is needed. ${ }^{16}$

Aspirin and clopidogrel are commonly used anti-platelet aggregation drugs in clinic, but their mechanisms of action are different. Therefore, the treatment of ACI with aspirin or clopidogrel alone has some limitations, while the effect of dual antiplatelet therapy is relatively definite. ${ }^{17,18}$ Butylphthalide is a new drug approved for market in 2004. It is a synthetic racemate, whose structure is similar to l-apigenin in celery seed. A relevant research shows that the use of butylphthalide in the treatment of stroke patients can block the inflammatory response of patients after cerebral ischemia and hypoxia from multiple channels and links and play an effective role in protecting and repairing patients' brain neurons. ${ }^{19}$ The main functions of butylphthalide are: 1) to improve the ischemic tissue of brain tissue, promote blood flow, and reduce the degree of brain injury; 2) to effectively resist platelet aggregation and thrombosis, relieve brain edema and ischemic area, promote blood microcirculation of brain tissue, improve its energy metabolism, and achieve the effect of inhibiting neuronal apoptosis; 3) to effectively increase the content of nitric oxide (NO) and prostacyclin (PGI2) in cerebral blood vessels, reduce the content of arachidonic acid and the secretion of glutamate, alleviate the phenomenon of intracellular calcium overload, improve the activity of antioxidant enzymes, and reduce and inhibit the inflammatory response in the injured area. ${ }^{20,21}$ The sequential therapy of butylphthalide refers to choosing different treatment methods at proper time according to different conditions of patients, including using different drugs/different ways of medication. For ACI patients in critical condition, intravenous administration of butylphthalide in the early stage can help to alleviate the patient's condition quickly; after two weeks of treatment, oral administration of butylphthalide cannot only maintain the effect of drug treatment, but also facilitate the treatment of patients at home after discharge. At present, butylphthalide has entered into phase IV clinical trials from phase I clinical trials, and its effectiveness and safety have been widely recognized. . $^{22}$

This study treated patients with progressive cerebral infarction using butylphthalide and 
sodium chloride injection in combination with dual anti-platelet therapy. The research results showed that the effective rate of treatment in the observation group was $92.5 \%$, significantly higher than that in the control group $(78.0 \%)$. There was significant difference between the two groups $(\mathrm{P}<0.05)$. NIHSS score in the observation group was significantly higher than that in the control group $(\mathrm{P}<0.05)$. The ADL score of the observation group was significantly lower than that of the control group, and the difference between the two groups was statistically significant $(\mathrm{P}<0.05)$. The above results showed that sequential treatment of butylphthalide could effectively promote the establishment of collateral circulation, improve the blood flow of ischemic penumbra, promote the recovery of nerve cells, improve the prognosis of patients and reduce the disability rate of cerebral infarction, which is similar to previous research results..$^{25}$

A recent study has shown that hydrogen sulfide can improve neurons during cerebral ischemia and hypoxia, ${ }^{26}$ and it is beneficial to alleviate cerebral ischemia-reperfusion injury. 3-MST is the main enzyme producing hydrogen sulfide in the nervous system, and its plasma level is related to the degree of brain injury. $A \beta_{42}$ is the main component of senile plaque, the brain ischemia and hypoxia can affect $A \beta_{42}$, and its plasma level is closely related to the degree of nerve injury. The results of the present study showed that the plasma level of 3-MST in the observation group was higher than that in the control group after treatment, and the plasma level of $A \beta_{42}$ was lower than that in the control group. It suggested that sequential butylphthalide therapy combined with dual antiplatelet therapy could effectively increase the content of hydrogen sulfide in elderly patients with $\mathrm{ACI}$ and reduce the content of $\mathrm{A} \beta_{42}$ to play a neuroprotective role and reduce neurotoxicity, which was consistent with Yang. ${ }^{27}$ In addition, no serious adverse reactions occurred during the treatment in both groups, suggesting that sequential butylphthalide therapy combined with dual antiplatelet therapy was safer for elderly ACI.

Limitations of the Study: The sample size of this study was small, and moreover this study was single-center. The long-term prognosis was not further observed. Therefore, the number of cases should be increased in the future, and multi-center, long-term observation study is needed for further explication.

\section{CONCLUSION}

Sequential butylphthalide therapy combined with dual antiplatelet therapy is effective in the treatment of elderly ACI. It can effectively improve the plasma level of 3-MST and decrease the plasma level of $A \beta_{42}$. It is conducive to improving the neurological function and living ability of patients and has high safety; therefore, it is worthy of clinical application. However, the sample size of this study is small, and the long-term prognosis was not further observed. Therefore, we should increase the number of cases in future studies and look forward to further clarification of multicenter and long-term observation studies.

\section{Declaration of interest: None.}

Grant Support \& Financial Disclosures: None.

\section{REFERENCES}

1. Wu W, Guan Y, Xu K, Fu, XJ, Lei XF, Lei LJ, et al. Plasma homocysteine levels predict the risk of acute cerebral infarction in patients with carotid artery lesions. Mol Neurobiol. 2016;53(4):2510-2517. doi: 10.1007/s12035015-9226-y

2. Liu J, Xing $Y$, Gao $Y$, Zhou C. Changes in serum interleukin-33 levels in patients with acute cerebral infarction. J Clin Neurosci. 2014;21(2):298-300. doi: 10.1016/j.jocn.2013.04.036

3. Sepp D, Franz D, Triftshaeuser N, Ott I, EspositoBauer L, Feurer R, et al. Mobilization of CD133+ progenitor cells in patients with acute cerebral infarction. PLoS One. 2014;9(3):e70796. doi: 10.1371/ journal.pone.0070796

4. Mansfield MW, Catto AJ, Carter AM, Grant PJ. Fibrinolytic measurements in type 2 diabetic patients with acute cerebral infarction. Diabetic Med. 1998;15(11):953-957. doi: 10.1002/(SICI)10969136(1998110)15:11<953::AID-DIA712>3.0.CO;2-6

5. Tada K, Uchida K, Kanayama H, Kajitani T, Yamamoto Y, Sanada S. Usefulness of iterative reconstruction method in the field of acute cerebral infarction computed tomography examination. Nihon Hoshasen Gijutsu Gakkai Zasshi. 2015;71(11):1090-1095. doi: 10.6009/jjrt.2015_JSRT_71.11.1090

6. Zhang X, Wu J, Zhang B. Xuesaitong injection as one adjuvant treatment of acute cerebral infarction: a systematic review and meta-analysis. BMC Complem Altern M. 2015;15(1):36. doi: 10.1186/ s12906-015-0560-4

7. Wu XN, Zhang T, Wang J, Liu XY, Li ZS, Xiang W, et al. Magnetic resonance diffusion tensor imaging following major ozonated autohemotherapy for treatment of acute cerebral infarction. Neural Regen Res. 2016;11(7):1115-1121. doi: 10.4103/16735374.187046

8. Nakamura Y, Nakajima H, Kimura F, Unoda K, Arawaka S. Preventive effect of cilostazol on pneumonia in patients with acute cerebral infarction. J Stroke Cerebrovasc Dis. 2018;27(9):2354-2359. doi: 10.1016/j. jstrokecerebrovasdis.2018.04.024 
9. Wang JP, He X, Zhang M, Yu H, Yang L, Meng T. Clinical study on probucol with different doses in the treatment of acute cerebral infarction. China Pharm. 2014;25(10):908910. doi: 10.6039/j.issn.1001-0408.2014.10.16

10. Zuo FT, Dong AQ. Dual antiplatelet aggregation for treatment of ischemic stroke. J Brain Nerv Dis. 2016;24(1):55-59.

11. Branch of Neurology of Chinese Medical Association. Cerebrovascular Group of Branch of Neurology of Chinese Medical Association. Guide for the diagnosis and treatment of acute ischemic stroke in China (2014). Chin J Neurol. 2015;48(4):246-257. doi: 10.3760/cma.j.is sn.1006-7876.2015.04.002

12. Wang $X$, Zhao $X$, Johnston SC, Xian $Y$, Hu B, Wang C, et al. Effect of clopidogrel with aspirin on functional outcome in TIA or minor stroke: CHANCE substudy. Neurology. 2015;85(7):573-579. doi: 10.1212/WNL.0000000000001844

13. Branch of Neurology of Chinese Medical Association. Cerebrovascular Group of Branch of Neurology of Chinese Medical Association. Guide for the secondary prevention for ischemic stroke and transient ischemic attack in China. Chin J Neurol. 2015;48(4):258-273. doi: 10.3760/cma.j.is sn.1006-7876.2015.04.003

14. Liu YJ. Clinical analysis of butylphthalide sequential therapy for acute cerebral infarction. Guide Chin Med. 2017;15(28):40-41.

15. Shi Z, Fu XL, Xia PS, Yuan WJ, Li SE, Zheng WC. Correlation analysis between thromboelastography and early neurological deterioration in patients with acute cerebral infarction. Chin J Cerebrovasc Dis. 2018;15(1):2630. doi: 10.3969/j.issn.1672-5921.2018.01.006

16. Akinseye OA, Shahreyar M, Heckle MR, Khouzam RN Simultaneous acute cardio-cerebral infarction: is there a consensus for management? Ann Translat Med. 2018;6(1):7. doi: $10.21037 / \mathrm{atm} .2017 .11 .06$

17. Fiolaki A, Katsanos AH, Kyritsis AP, Papadaki S, Kosmidou M, Moschonas IC, et al. High on treatment platelet reactivity to aspirin and clopidogrel in ischemic stroke: A systematic review and meta-analysis. J Neurol Sci. 2017;376:112-116. doi: 10.1016/j.jns.2017.03.010

18. Milionis HJ, Gerotziafas G, Kostapanos MS, Vemmou A, Zis $\mathrm{P}$, Spengos $\mathrm{K}$, et al. Clopidogrel vs. aspirin treatment on admission improves 5-year survival after a first-ever acute ischemic stroke. data from the Athens Stroke Outcome Project. Arch Med Res. 2011;42(6):443-450. doi: 10.1016/j.arcmed.2011.09.001

19. Feng YP, Xu HL. The search for new drugs for the treatment of stroke. Pharm News. 2007;7:26.

20. Li Y, Lu Z, Keog H, Yu SP, Wei L. Erythropoietin-induced neurovas-cular protection, angiogenesis, and cerebral blood flow restoration after focal ischemia in mice. Cereb Blood Flow Matab. 2007;27(5):1043-1054. doi: 10.1038/ sj.jcbfm. 9600417
21. Li GQ, WU JH, Yang XX, Hong ZQ. Effects of dl-3nbutylphthalide on the expression of apoptosis-related factors in rats with focal cerebral ischemia-reperfusion. Chin J Clin Pharmacol. 2011;27(9):682-685.

22. Li J. Effects of butylphthalide sequential therapy on neurological function in patients with transient cerebral ischemia. Chin J Pract Nerv Dis. 2015;18(22):135-136. doi: 10.3969/j.issn.1673-5110.2015.22.100

23. Ji Z, Fan XB, He Y. Impact of Butylphthalide combined with Oxiracetam on neurological function, cognitive function and quality of life of patients with cerebral infarctioninduced cognitive disorders. Pract J Cardiac Cereb Pneum Vasc Dis. 2016;24(5):43-46.

24. Kong L, Shao HY. Clinical observation of butylphthalide injection in the treatment of progressive cerebral infarction. Chin Pract Med. 2016;11(11):139-140.

25. Tang SC, Luo CJ, Zhang KH, Li K, Fan XH, Ning LP, et al. Effects of dl-3-n-butylphthalide on serum VEGF and bFGF levels in acute cerebral infarction. Eur Rev Med Pharmacol Sci. 2017;21(19):4431-4436.

26. Yin W, Lan L, Huang Z, Ji J, Fang J, Wang X, et al. Discovery of a ring-opened derivative of 3-n- butylphthalide bearing $\mathrm{NO} / \mathrm{H} 2 \mathrm{~S}$ - donating moieties as a potential anti-ischemic stroke agent. Eur J Med Chem. 2016;115:369-380. doi: 10.1016/j.ejmech.2016.03.044

27. Yang GF, Gao Y, Wang W, Ji JG, Li XF, Zhang RL, Xu L. Comparative proteomics study of dl-3-n-butylphthalide protecting PC12 cells from hypoxia-induced damage. Chin J Neuromed. 2013;12(3):251-255. doi: 10.3760/cma.j. issn.1671-8925.2013.03.008

\section{Authors' Contribution:}

XWW, JYH \& ZGW: Study design, data collection and analysis.

XWW, JJW, XMW \& XYL: Manuscript preparation, drafting and revising.

XYL \& ZGW: Review and final approval of manuscript.

XWW: Responsible and accountable for the accuracy or integrity of the work.
Authors:

1. Xuewen Wo

2. Jinyan Han

3. Jiajia Wang

4. Xinmin Wang

5. Xiaoying Liu

6. Zhonggong Wang

1-6: Departments of Neurology, Binzhou People's Hospital, Shandong, 256610, China. 